\title{
Comunicación hipermediática y jóvenes
}

\section{Hypermedia communication and young people}

Jorge Alberto Hidalgo Toledo*

Universidad Anáhuac México

Av. Universidad Anáhuac núm. 46, Col. Lomas Anáhuac, Huixquilucan, Estado de México, C.P. 052786, México

\section{Luis Felipe Ramírez Alvarado**}

Universidad Anáhuac México

Av. Universidad Anáhuac núm. 46, Col. Lomas Anáhuac,

Huixquilucan, Estado de México, C.P. 052786, México jhidalgo@anahuac.mx

https://orcid.org/0000-0002-6204-9534

\section{Editor: Rogelio del Prado Flores}

Fecha de recepción: 22 de junio de 2021

https://doi.org/10.36105/stx.2021edesp50aniv.05

Fecha de aceptación: 25 de agosto de 2021

* Investigador del CICA, Universidad Anáhuac México. Doctor en Comunicación Aplicada por la Universidad Anáhuac. Coordinador académico de Posgrados de la Facultad de Comunicación de la Universidad Anáhuac México. Expresidente del CONEICC. Expresidente de la AMIC, vicepresidente de Asuntos Educativos de la Academia Mexicana de la Comunicación y secretario de la Asociación de Comunicadores Católicos Signis México. Coautor de Transformaciones mediáticas y comunicacionales en la era posdigital (202I), Medios y mediaciones en la cultura digital (2017), Signo vital: comunicación estratégica en la promoción de la salud (2011) y Comunicación Masiva en Hispanoamérica: Cultura y literatura mediática (2005).

** Investigador del CICA, Universidad Anáhuac México. Doctor en Estudios Humanísticos por el Tecnológico de Monterrey, Campus Ciudad de México. Coordinador de la Licenciatura en Comunicación para la Facultad de Comunicación de la Universidad Anáhuac México, Campus Sur. Profesor invitado por la Universidad de Savoie Mont-Blanc, Francia para el Master en Création Numérique. Coautor de Phenomenological perspectives on the communication modalities of 360 video (2020); The visual analysis of urban realities trough 360 video: The $19 S$ earthquake case in México and the Project SI9 (2019); The visual representation of the hologram: a comparative approach to its political and social uses (2018). The Performance of Innovation Systems for the Cases of Mexico and Korea in the Context of Knowledge Societies (2015). 


\section{RESUMEN}

El texto describe a las jóvenes audiencias en el contexto mexicano; el rol que juegan los hipermedios en sus vidas y las implicaciones sociales, económicas, culturales y políticas que derivan de la mediatización de la vida y la cultura. Los jóvenes viven un momento particular de la historia que los ha llevado a establecer vínculos más profundos con los medios, mismos que se han vuelto el punto de convergencia entre las culturas juveniles, la cultura popular, las prácticas identitarias, los escenarios donde se amplifica la vida urbana y su corporalidad. Esta investigación da cuenta de la condición juvenil para situarla en el contexto sociocultural, dado que la noción de juventud en sí misma es una construcción social relacional. Por tanto, este texto sirve de cartografía a los lectores para navegar por las distintas culturas, prácticas y formas de autorreconocimiento y heterorreconocimiento de las culturas juveniles y sus consumos mediáticos particulares.

Palabras clave: comunicación, hipermediación, consumo, audiencias, cultura, significación.

\section{ABSTRACT}

This text describes young audiences in the Mexican context; the role that hypermedia plays today in their lives and the social, economic, cultural and political implications that derive from the mediatization of life and culture. Young people, as can be seen throughout the many studies that have been carried out at the international level, live in a particular moment in history that has led them to establish deeper links with the media, which have become the point of convergence between youth cultures, popular culture, identity practices, scenarios where urban life and its corporality are amplified. This research aims to account for the youth condition to place it in the sociocultural context, given that the notion of youth is a relational social construction. Therefore, this text serves as a cartography for readers to navigate through the different cultures, practices, and forms of self-recognition and heterorecognition of youth cultures and their media consumption.

Keywords: communication, hypermediation, consumption, audiences, culture, significance. 


\title{
INTRODUCCIÓN
}

\begin{abstract}
Muchos jóvenes no están en contacto con medios e hipermedios. De los que sí lo están creo que hay una correlación entre múltiples variables históricas, políticas, culturales, económicas, tanto del entorno como del sujeto que configuran las prácticas sociales, los imaginarios y las representaciones que tienen los jóvenes en relación a los medios e hipermedios. Lo que sí me queda claro es que el mercado se preocupa por hacer creer una serie de discursos que hacen que algunos sujetos piensen que el papel de los medios ES de determinada manera y luego vienen los contradiscursos y la resistencia y las otras realidades... para mí es un tema complejo en donde es muy difícil generalizar.
\end{abstract}

ENTREVISTA A EXPERTOS ${ }^{\mathrm{E}}$ EAEI, CIUDAD DE MÉXICO, MÉXICO

La comunicación y los procesos de mediación siempre han estado en el corazón de la vida de las personas. Los medios de comunicación, desde sus orígenes, permitieron extender la memoria del hombre, resguardar su historia, expandir su representación, reproducir sus ideas, ubicarlo en el mundo, permitirle llegar a todos los rincones del mundo. Hoy los medios de comunicación ante la convergencia digital se han convertido en el principal puente de significación. Desde ellos las personas comprenden la realidad, profundizan en su entorno, en síntesis, le dan cuerpo y sustancia a gran parte de sus experiencias humanas. Los medios e hipermedios se han convertido en un territorio de significación permanente desde el cual los sujetos ya no solo captan mensajes, sino que desde ellos resignifican la vida.

Los medios e hipermedios son código, lenguaje, canal, narración y vehículo de la existencia. Les permiten conectar con el mundo, cuestionar los sistemas económicos, culturales, políticos y sociales; desde ellos socializan referencias cardinales.

El contexto comunicativo es el centro de la interacción humana en la hipermodernidad. La vida se ha hipermediatizado. Todas las experiencias significativas están siendo mediatizadas. Es por ello que se hace fundamental comprender cómo es que los medios construyen, difunden y comercializan dichas experiencias.

Esta dinámica se potencializó desde mediados del siglo pasado. No fue hasta que se dio el entrecruce entre las prácticas de consumo y el desarrollo de los teen media (cine, radio, te-

I Los Expertos (EaE) e Informantes Sociodigitales (SISD) citados en el presente trabajo participaron en un foro digital de discusión situado dentro de Facebook con la intención explícita de colaborar en el estudio indicando las motivaciones detrás de la pregunta: ¿Qué relación guardan los jóvenes con los nuevos medios? Aun cuando se tiene el consentimiento de los participantes se decidió guardar su anonimato. 
levisión, cómics, revistas, videojuegos, aplicaciones móviles...) que empezó a preocupar el carácter vulnerable de las jóvenes audiencias. Desde entonces preocupó el papel que desempeñaron los medios de comunicación como su principal pasatiempo, ya que los empezaron a consumir en todo momento, lugar y contexto.

Los medios e hipermedios, por tanto, se han convertido en el principal recurso expresivo y comunicacional de las culturas juveniles, tanto así que desde la década de los ochenta del siglo pasado se les han agrupado en cohortes generacionales tales como la Mtv Generation, la Generación Click, la Generación Net, la Generación (M)ediática, la Generación Nintendo, la Generación Txt, la Generación Mi Medio, Generación Hashtag y Generación Transmedia.

El presente texto intenta describir a las jóvenes audiencias en el contexto mexicano; el rol que juegan hoy los hipermedios en sus vidas y las implicaciones sociales, económicas, culturales y políticas que derivan de la digitalización y mediatización de la vida y la cultura.

\section{DESARROLLO. EL JOVEN Y LA NUEVA ECOLOGÍA DE MEDIOS COMO OBJETO DE ESTUDIO}

Los jóvenes hemos adoptado un estilo de vida a partir de la tecnología. Los medios tradicionales convergen con el internet y es así como el disfrute y la consulta de información se volvieron atemporales. Por eso influyen en abastecer de información, formar cultura y opinión, además de entretener.

SUJETO INFORMANTE SOCIO DIGITAL 87 , CIUDAD DE MÉXICO, MÉXICO

Desde los inicios de la investigación en comunicación ha estado presente el modo de recepción y consumo mediático de las jóvenes generaciones. Dichos estudios han revisado aspectos tales como: I) tiempo de consumo y desplazamiento de la autoridad de instituciones como la familia, la escuela y la iglesia (Silverstone, I994); 2) los medios como pasatiempo significativo (Watkins, 2009); 3) recurso de expresión y comunicación cultural (Buckingham, 2005); 4) vías de participación activa en la vida pública (Umaschi Bers, 2008); 5) amplificación de la cultura juvenil (Schor, 2006); 6) inevitables y omnipresentes procesos de comunicación, interacción y socialización (David, r999); 7) rutinas de la vida cotidiana y la cultura (Poniewozik, 2005);8) recursos simbólicos usados para distinguir e interpretar 
relaciones y definir identidades (Buckingham, 2008); 9) medios y sentido (Silverstone, 2004); y IO) promoción de actitudes o formas de conducta violentas, sexistas y racistas (Daniels, 2008); II) representaciones, prácticas y estéticas; I2) identidades hipermediales (Hidalgo, 2019); I3) El espacio social juvenil ${ }^{2}$ (Urteaga, 2010).

El joven como campo social y académico se ha hecho visible desde el siglo pasado por su participación activa en los espacios políticos, sociales, culturales, económicos y mediáticos. Se han estudiado las diferentes culturas urbanas como los beat, hippies, estudiantiles, la onda, punks, yuppies, trashers, ciberpunks, grunge, skatos, darketos, emos, góticos, technos, tribales, las tribus del pulgar, las generaciones Baby boomer, Gen X, Gen Net, Gen Media, Tweens, Millennials y Centennials.

Las investigaciones en este sentido han intentado dar cuenta de la relevancia que tienen los trabajos que estudian las implicaciones sociales de los medios de comunicación. Particularmente podemos destacar que existen en todos ellos: I) una preocupación por los derechos de los niños y jóvenes; 2) la manera como los contenidos violentos en los medios los convierten en audiencias vulnerables; 3 ) la exploración que hacen los usuarios en los medios para configurar sus identidades; 4) las visiones del mundo que aportan, así como las formas de ser y comportarse en él; 5) el uso de los medios como interfaces liberadoras; 6) la promoción de un pensamiento crítico que posibilite el empoderamiento y cambio social; 7) las prácticas de consumo cultural; 8) las interacciones y formas de socialización; 9) los fenómenos transversales como globalización, migración y digitalización de la cultura; Io) el uso de los medios en los procesos educativos y alfabetizadores; iI) el uso que hacen de los medios digitales para compartir ideas. Todos estos abordajes se han realizado desde la antropología, la sociología, la comunicación, la historia, la educación, la psicología social, las neurociencias, las ciencias de datos y las humanidades digitales. El abordaje de esta propuesta de investigación va desde la perspectiva teórica de la Nueva Ecología de Medios33.

Los principales ejes de trabajo de los estudios se centran en: lo Urbano, mediático y corporal (2008) y cuatro apartados de agregaciones juveniles como lo señala Maritza Urteaga: las bandas, el rock, los medios de comunicación y escenarios otros, entendidos éstos como el escolar, religioso y laboral (2000). Las principales tendencias de estudio analizan

2 Como recorte del campo del mundo social planteado por Bourdieu como espacio social. Es conceptualizado por Maritza Urteaga como "la base de principios de diferenciación o distribución constituido por el conjunto de propiedades que actúan en el universo social en cuestión, es decir propiedades capaces de conferir a quien las posea con fuerza, poder, en ese universo" (Urteaga Castro-Pozo, 20Io, p. 32).

3 Posición teórica que estudia a los medios de comunicación y las tecnologías de mediación y su relación socio-ambiental. Centran su atención en los ambientes en los que los medios interactúan con las personas. Sus principales contribuyentes fueron Marshall McLuhan, Neil Postman, Walter Ong y Lance Strate, entre otros. 
la integración de los jóvenes en las prácticas de consumo cultural y la irrupción del espacio urbano (Reguillo, 2000).

Este trabajo considera para su estudio las dimensiones de conexión, desconexión y mediatización. Esto debido a que los medios les permiten a los jóvenes, acceder, poseer, usar y acumular distintos capitales (ya sean cognitivos, sociales, políticos, culturales y simbólicos).

Ser joven implica el cambio social, la movilización, la negociación, la apropiación simbólica de los espacios y las instituciones. De ahí la importancia de comprender a los medios como ese espacio social juvenil como señala Maritza Urteaga:

Dentro del espacio social juvenil, los medios y las nuevas tecnologías no son simplemente mercancías simbólicas o marcadores de distinción, sino redes cruciales en la definición y distribución del conocimiento cultural. La diferencia entre estar dentro (in) o fuera (out) de la moda, alto o bajo en capital subcultural, se correlaciona de maneras complejas con grados de información, creación y exposición a los mismos. (20II, p.34)

\section{EL CONSUMO DE MEDIOS Y LOS JÓVENES}

Los hipermedios no determinan mi relación con los demás haciéndola mejor o peor. Creo que esta depende de la personalidad de los sujetos y de su entorno. Los medios son herramientas que en todo caso amplían la relación con el otro.

SUJETO INFORMANTE SOCIO DIGITAL SID2O, CIUDAD DE MÉXICO, MÉXICO

En México, como en gran parte del mundo se está viviendo un gran cambio en los estándares de uso y consumo mediático. Cifras publicadas por el estudio 15 Estudio sobre los Hábitos de los usuarios de Internet en México 2018 (2019) reportan la existencia de 82.7 millones de internautas que representan $7 \mathrm{I} \%$ de penetración nacional en personas mayores de seis años. El crecimiento en el uso de teléfonos inteligentes con acceso a Internet ha sido exponencial, hoy representa $92 \%$ de las conexiones versus $76 \%$ de una laptop. Es importante saber que el $84 \%$ de las personas ingresan desde sus hogares, $7 \%$ desde un café internet, $42 \%$ desde sus trabajos, $8 \%$ desde su lugar de estudio y $22 \%$ desde un lugar público; $51 \%$ de los internautas son mujeres y $50 \%$ del total de usuarios pertenecen a los niveles $\mathrm{ABC}+{ }^{4}$. La composición

${ }^{4}$ Los segmentos de mayor crecimiento $(+5 \%)$ han sido los $\mathrm{D}+\mathrm{y} \mathrm{D} / \mathrm{E}$. La distribución poblacional es la 
de edad del universo de usuarios registra $26 \%$ de los jóvenes mexicanos entre los 6 y los 17 años de edad.

En función del presente texto, la composición del universo de internautas por edad destaca lo siguiente: I $4 \%$ entre los I2 y I7 años; I $8 \%$ entre los I 8 y $24 ; 22 \%$ entre los 25 y $34 \%$. Así se tiene que $32 \%$ de los jóvenes mexicanos entre i2 y 24 años están en Internet; viviendo $25 \%$ en el Centro Sur del país. Las actividades realizadas en línea oscilan entre $82 \%$, acceder a redes sociales; $77 \%$, enviar y recibir mails; $78 \%$, chatear; $76 \%$, buscar información; $68 \%$, utilizar mapas; $63 \%$, escuchar música; $59 \%$, leer, ver y escuchar contenidos relevantes; $65 \%$, ver películas en streaming; $42 \%$, estudiar en línea; $51 \%$, hacer videollamadas; $28 \%$, jugar en línea.

En promedio los navegantes invierten 8 horas 20 minutos para consultar información de lunes a viernes conectándose en promedio entre dos y tres veces por semana, esto representa ocho minutos más que en 2018. Sus principales actividades son: ocio $(56 \%)$; finanzas (54\%); formación (46\%); trámites (44\%); compras (46\%); contactos $(42 \%)$. De los rubros anteriores, destaca un crecimiento en la información relativa a finanzas con un $8 \%$ más que en 2018 , y el área de contactos con $21 \%$ respecto a 2017 .

Vale la pena destacar algunos consumos de contenidos hipermediáticos que han tenido un crecimiento significativo en el último año. En este sentido, las películas y su distribución en plataformas en línea se encuentran en $52 \%$, con un crecimiento de siete puntos porcentuales respecto al 20I8. Del mismo modo, el consumo de servicios como alimentos tuvo un crecimiento de $11 \%$ y el de servicios financieros con $21 \%$ más que el año anterior. Esto último puede explicarse por la adopción de las herramientas digitales como la banca en línea, misma que permite el acceso a diferentes productos financieros no solo en vía equipo de cómputo, sino por el uso de las aplicaciones móviles para las finanzas personales. Por otra parte, la publicidad parece sufrir un revés año con año, pues solo $22 \%$ de los internautas interactúan con publicidad que ven en línea, lo que representa menos que en 2018.

En términos de las redes sociales, el internauta promedio se encuentra inscrito en al menos cinco redes. Destaca en la punta el uso de Facebook y la continua caída de Twitter en el ranking, este año con Io\% menos respecto del año anterior. Aunque pareciera poco significativo, los usuarios de internet han incrementado el tiempo de uso de redes sociales en dos minutos respecto al año anterior, esto para un total de 3 horas y 3 Iminutos. Este acceso se da, primordialmente por el teléfono inteligente.

siguiente: $\mathrm{ABC}+40 \%, \mathrm{C} 20 \%, \mathrm{D}+27 \%$ y D/E I4\%. Lo cual habla de una mayor democratización del uso y consumo de Internet. 


\section{LAS CIBERCULTURAS JUVENILES MEXICANAS}

Los medios e hipermedios juegan un papel muy importante en la vida de los jóvenes, pues se relacionan con gente de su edad, siembran amistades, intercambian conocimientos, se acercan a otras culturas

SUJETO INFORMANTE SOCIO DIGITAL SID69, CELAYA, GUANAJUATO, MÉXICO

De acuerdo con los principales resultados de la Encuesta Intercensal 2015: Estados Unidos Mexicanos (Inegi, 2015), se redujo la población infantil y adolescentes (o a I4 años) de $34.2 \%$ a $27.4 \%$; de jóvenes ( 15 a 29 años) de $28.5 \%$ a $27 \%$; mientras que de adultos jóvenes (30 a 44 años) creció de I9.6\% a 21\%; y de adultos ( 45 a 59 años) de I0.5\% a I2.2\%. Esto es una tendencia creciente y que se verá reflejada en el acceso a la educación, al consumo de medios, apropiación de tecnologías de información y modos de socialización a través de estas herramientas tecnológicas.

En un lapso de Io y 15 años esa gran porción de la población en edad infantil y juvenil serán los que tendrán en sus manos las posibilidades de cambio y desarrollo del país. Su vinculación con los medios y los contenidos que de ellos perciben ¿̇les estarán permitiendo formar una identidad y formas culturales que les permitan contribuir pro socialmente en el complejo entramado social, económico e institucional que está conformando?

Los medios, como prolongación esencial de la vida, deben ser juzgados no con un afán condenatorio, sino con miras a desentrañar sus limitaciones y posibilidades. Si los jóvenes hoy día emplean los medios para entender cómo es su sociedad, extraer opiniones sobre los diversos aspectos de la vida y construir con ello su propio yo (Montero, 2006), habrá que plantearse los cuestionamientos éticos que deriven de las interacciones mediáticas y sociales y ver cómo aprovecharlos más que para seguir construyendo consumidores, para formar personas íntegras que puedan contribuir al desarrollo del país.

CONCLUSIÓN

Los jóvenes socializan y se divierten. Los hipermedios son parte ya del imaginario del adolescente urbano. NO hay que perder de vista que a veces los padres promueven el uso para estar en contacto con ellos, porque trabajan porque hay escaso tiempo de estar con ellos. 
Los medios cambiaron al mundo y con ello cambiaron también a las audiencias. Los estudios de consumo mediático dejan ver cómo desde sus inicios los estudios de comunicación notaron el impacto que estaban teniendo los medios en la manera como se comprendían y vivían los derechos de los niños y jóvenes. En su condición de inmadurez biológica e intelectual se les contempló como audiencias vulnerables y altamente expuestas a contenidos cargados de violencia, lenguaje explícito y promotores de actividades propias de la vida adulta. De esta visión proteccionista se pasó al estudio de la relación de los medios como articuladores de la realidad social.

Crecer y vivir en un mundo saturado de medios que aportan visiones del mundo y formas de ser, estar y comportarse en él es la naturaleza de las personas desde mitad del siglo pasado. Los medios, como experiencias vitales y puertas de acceso al mundo para los jóvenes, se han convertido en interfaces que les permiten participar en los procesos sociales, económicos, culturales e ideológicos.

Este texto registró la telemediatización y conectividad global que están viviendo las audiencias y la urgencia que existe por formar en los usuarios pensamiento crítico, activo y propositivo de tal forma que puedan participar, comunicarse, interactuar, socializar en un contexto reticular y global.

Los jóvenes, como se pudo ver a lo largo de los múltiples estudios que se han realizado a nivel internacional, están viviendo en un momento particular de la historia en que el individualismo, consumismo y crisis de las instituciones los ha llevado a establecer vínculos más profundos con los medios. Los medios se han vuelto el punto de convergencia entre las culturas juveniles, la cultura popular, las prácticas identitarias, los escenarios donde se amplifica la vida urbana y su corporalidad.

Esta investigación particularmente pretendió dar cuenta de la condición juvenil más allá de la dimensión biológica y psicológica para situarla en el contexto sociocultural, dado que la noción de juventud en sí misma es una construcción social relacional. Por tanto, este apartado sirve de cartografía a los lectores para navegar por las distintas culturas, prácticas y formas de autorreconocimiento y heterorreconocimiento de las culturas juveniles y sus consumos mediáticos particulares.

En este sentido, se ha situado en este texto una dimensión más para comprender al joven en el campo social: la mediatizada. La condición mediatizada se refiere a los jóvenes que están permanentemente estructurando y reconfigurando sus prácticas sociales y culturales desde la dimensión mediática.

Los medios han dotado a los jóvenes de distintos capitales que les permiten acceder, poseer, usar y acumular capitales políticos, culturales, sociales y simbólicos. En ese proceso de mediación, lo joven se torna una metáfora del cambio social, de la movilización, de la ne- 
gociación de las nuevas prácticas culturales, estéticas y simbólicas. De la apropiación intangible de espacios y territorios en los que trazan nuevos itinerarios y rutas de significación.

Si los medios son el espacio social juvenil, donde se estructura y articula la configuración de la identidad de las audiencias, es importante comprender la manera como se está constituyendo el campo, la manera como se establecen las prácticas, distinciones, gustos, significaciones y actitudes.

Los medios como marcadores y redes de definición de los individuos concentran la dimensión comunicativa, creativa, espacial y expresiva de las personas. Existe, pues, una nueva ecología donde medios y tecnología están cambiando la vida de las personas y reconfigurando al mismo mundo.

Los medios y las TIC como herramientas para moldear y modificar la realidad nos presentan un nuevo horizonte con retos, riesgos y oportunidades. Temas como inclusión, exclusión, hibridez, aprendizaje, competencias digitales, participación cívica, desarrollo, autorregulación, libertad de expresión, conexión y juego se incorporan en la agenda de estudio y análisis de esta nueva condición del ser en la era digital.

Las tecnologías de información y comunicación y su acelerado proceso evolutivo y de incorporación en la vida y los contextos dislocaron por completo nuestras experiencias temporales, espaciales, cognitivas y existenciales. Su capacidad para envolver y amplificar la vida, sedujeron a los usuarios. Los llevaron a pasar de una condición pasiva a otra más activa. Hoy las nuevas generaciones mediáticas procesan información y comprometen sus talentos con los nuevos medios y dispositivos. Desde ellos exploran normas, hábitos, costumbres y nuevas amistades.

Dada esta nueva realidad se ha constituido una cultura bipermediática, con nuevas tipologías de audiencias, agencias y babitus. El espacio mediático es el espacio del ser, es el lugar donde el yo se presenta en modo híbrido, de forma atemporal, instantánea, hiperconectado, deslocalizado, inmaterialy soportado por la gravidez simbólica que permite su codificación.

La mediósfera (Debray, 200I), la mediópolis (Silverstone, 2004), la tecnópolis (Postman, 1994), la ciberpolis (Zygmunt, 1998) no son otra cosa que ese espacio híbrido que se tiende entre la realidad física, la virtual y la mental. Son el soporte que conecta todos los espacios y que permiten al individuo incrustarse de modo natural en ese horizonte de estructuras y ambientes simbólicos donde es posible vivir, trabajar, moverse, ejercitarse y divirtiese en cuerpo y mente en varios espacios a la vez.

Hoy estamos ante ciberculturas juveniles que extienden sus prácticas y representaciones en los entornos bipermediales. Vida y pantallas engranaras; socialidades, tecnicidades y subjetividad en red se cuestionan y construyen sentidos cada vez que se conectan, instalan, encuentran y estructuran en estos contenedores simbólicos. Hoy los jóvenes están moldean- 
do sus identidades individuales y colectivas en relación con los otros desde los medios; ahí establecen significados sociales, formas de identificación-diferenciación social y consolidación de la cultura-mundo.

Esta nueva forma de sociabilidad, percepción y sensibilidad desde las pantallas implica nuevos abordajes territoriales de las culturas juveniles. Por ello en este texto se hicieron notorias las nuevas genealogías, subculturas, agendas, identificaciones y diferenciaciones de las performatividades de lo joven. Jóvenes, medios y cultura son la triada que está detonando una nueva revolución simbólica/antropológica que nos obliga a repensar las preguntas básicas alrededor de la persona y su lugar en el mundo.

\section{REFERENCIAS}

Asociación deInternet Mx.(2019).15. ${ }^{\circ}$ Estudio sobrelosHábitos delos Usuarios de Internet en México 2019. https://www.asociaciondeinternet.mx/es/component/remository/Habitos-de-Internet/I5-Estudio-sobre-los-Habitos-de-los-Usuarios-de-Internet-en-Mexico-20Ig-version-publica/lang,es-es/?Itemid=

Buckingham, D. (2005). Educación en medios: Alfabetización, aprendizaje y cultura contemporánea . Paidós Ibérica.

Buckingham, D. (2008). Más allá de la tecnología. Aprendizaje infantil en la era de la cultura digital. Manantial.

Daniels, J. (2008). Race, civil rights, and hate speech in the digital era. En A. Everett, Learning Race and Etbnicity: Youtb and Digital Media (págs. 129-155). The MIT Press.

David, P. (1999). Children's Access to Media and Media Use. En C. Von Feilitzen, \& U. Carlsson, Cbildren and Media. Image Education Participation. Yearbook 1999 . Nordicom.

Debray, R. (200I). Introducción a la mediología. Paidós.

Fondo de Población de las Naciones Unidas en México. (20II). Marco de acción sobre adolescentes y jóvenes. http://www.unfpa.org.mx/estrategia_jovenes.php

Gómez,H. (2008). Estratos espaciales y de comunicación en los estudios sobre la juventud. Una revisión de los estudios de los consumos culturales juveniles en México. En M. A. Rebeil Corella, XV Anuario de Investigación de la Comunicación, CONEICC (págs. I85-214). CONEICC; Universidad Anáhuac; Universidad Mayab; Universidad Iberoamericana León; Universidad Autónoma de San Luis Potosí; Tecnológico de Monterrey.

Hidalgo, J. (20II). I-dentidad hipermedial: nuevos medios, nuevas audiencias, ¿nuevas identidades? Revista del Centro de Investigación. Universidad La Salle, 9(35), 5-Io. http://www.redalyc.org/ pdf $/ 342 / 34218346002 . p d f$ 
Hidalgo, J. (20I9). Jóvenes hipermediales: hipermediatización del Yo y las juventudes hiperconectadas. En D. Barredo Ibáñez, M. Rodrigues da Cunha, y J. Hidalgo Toledo, Fóvenes, participación y medios de comunicación digitales en América Latin (págs. 17-46). Cuadernos Artesanales de Comunicación.

Inegi. (20II). Estadísticas de Población, Hogares y Vivienda. Distribución por edad y sexo. Instituto Nacional de Estadística y Geografía.

Inegi. (20I5). Principales resultados de la Encuesta Intercensal 20I5: Estados Unidos Mexicanos. Insituto Nacional de Estadística y Geografía.

Instituto Mexicano de la Juventud. (20II). Encuesta Nacional de Juventud 20Io. http://www.imjuventud.gob.mx/imgs/uploads/Presentacion_ENJ_20IO_Dr_Tuiran_V4am.pdf

Kirsh, S. J. (2010). Media and youth: A developmental perspective. Wiley-Blackwell.

Manovich, L. (2012). El lenguaje de los nuevos medios de comunicación: la imagen en la era digital. Paidós.

Mendoza, M. E., y Tapia Colocia, G. (2011). Situación demográfica de México I9Io-I920. (F. d. México, Productor).www.unfpa.org.mx/publicaciones/cuadro_4.pdf

Montero, Y. (2006). Televisión, valores y adolescencia. Gedisa.

Poniewozik, J. (2005). The big fat year in culture. En R. B. Browne, Profiles of popular culture: a reader (págs. 376-388). The University of Wisconsin Press.

Postman, N. (1994). Tecnopolis: la rendición de la cultura a la tecnología. Galaxia Gutenberg.

Reguillo, R. (2000). Las culturas juveniles: un campo de estudio. Breve agenda para la discusión. En G., Medina, Aproximaciones a la diversidad juvenil. El Colegio de México.

Schor, J. (2006). Nacidos para comprar: los nuevos consumidores infantiles. Paidós.

Silverstone, R. (1994). Televisión y vida cotidiana. Amorrortu Editores.

Silverstone, R. (2004). ¿Por qué estudiar los medios? Amorrortu Editores.

Strauss, W., y Howe, N. (I99I). Generations: The bistory of America's Future, 1584 to 2069. Harper Perennial.

Umaschi Bers, M. (2008). Civic identities, online technologies: from designing civics curriculum to supporting civic experiences. En W. L. Bennett, Civic Life Online: Learning How Digital Media Can Engage Youth (pp. 139-160). The MIT Press.

Urteaga, M. (2000). Formas de agregación juvenil. En A. Pérez Islas, Fóvenes: Una evaluación del conocimiento. La investigación sobre la juventud en México 1986-1999. Instituto Mexicano de la Juventud; Centro de Investigación y Estudios sobre la Juventud.

Urteaga, M. (20I0). Género, clase y etnia. Los modos de ser joven. En R. Reguillo, Los jóvenes en México (págs. 15-5I). Fondo de Cultura Económica; Conaculta. 
Urteaga, M. (2011). La construcción juvenil de la realidad: jóvenes mexicanos contemporáneos. Universidad Autónoma Metropolitana; Juan Pablos Editor.

Watkins, S. C. (2009). The young \& the digital: What the migration to social-network sites, games, and anytime, anywhere media means for our futur. Beacon Press.

Zygmunt, B. (1998). Globalization: The Human Consequences. Polity.

Esta obra está bajo Licencia Creative Commons Atribución-NoComercial-SinDerivadas 4.o Internacional. 